\title{
1 Significant organic carbon acquisition by Prochlorococcus in the
}

\section{oceans}

3

4 Zhen Wu \#, Dikla Aharonovich²\#, Dalit Roth-Rosenberg ${ }^{2}$, Osnat Weissberg², Tal Luzzatto-Knaan²,

5 Angela Vogts ${ }^{3}$, Luca Zoccarato ${ }^{4}$, Falk Eigemann ${ }^{3}$, Hans-Peter Grossartt ${ }^{4,5}$, Maren Voss ${ }^{3}$, Michael J.

6 Follows $^{* 1}$ and Daniel Sher *2

7

$8{ }^{1}$ Department of Earth, Atmospheric and Planetary Sciences, Massachusetts Institute of

9 Technology, Cambridge, MA 02139, USA

10 'Department of Marine Biology, Leon H. Charney School of Marine Sciences, University of Haifa,

1131905 , Israel

$12{ }^{3}$ Leibniz-Institute for Baltic Sea Research, Seestrasse 15, D-18119 Warnemuende, Germany

$13{ }^{4}$ Department of Experimental Limnology, Leibniz-Institute of Freshwater Ecology and Inland

14 Fisheries, Alte Fischerhuette 2, D-16775 Stechlin, Germany

$15{ }^{5}$ Potsdam University, Institute of Biochemistry and Biology, Maulbeeralle 2, D-14469 Potsdam,

16 Germany

17

18 * Corresponding authors: mick@ocean.mit.edu, dsher@univ.haifa.ac.il

19 \# These authors contributed equally to this study

20 


\section{Abstract}

23 Marine phytoplankton are responsible for about half of the photosynthesis on Earth. Many are

24 mixotrophs, combining photosynthesis with heterotrophic assimilation of organic carbon but the

25 relative contribution of these two carbon sources is not well quantified. Here, single-cell

26 measurements reveal that Prochlorococcus at the base of the photic zone in the Eastern

27 Mediterranean Sea are obtaining only $\sim 20 \%$ of carbon required for growth by photosynthesis.

28 Consistently, laboratory-calibrated evaluations of Prochlorococcus photosynthesis indicate that

29 carbon fixation is systematically too low to support published in situ growth rates in the deep

30 photic layer of the Pacific Ocean. Furthermore, agent-based model simulations show that

31 mixotrophic cells maintain realistic growth rates and populations 10s of meters deeper than

32 obligate photo-autotrophs, deepening the nutricline and Deep Chlorophyll Maximum by 20 m.

33 Time-series of Prochlorococcus ecotype-abundance from the subtropical North Atlantic and North

34 Pacific suggest that up to $30 \%$ of the Prochlorococcus cells live where light intensity is not enough

35 to sustain obligate photo-autotrophic populations during warm, stratified periods. Together,

36 these data and models suggest that mixotrophy underpins the ecological success of a large

37 fraction of the global Prochlorococcus population and its collective genetic diversity. 
Photosynthesis by phytoplankton provides most of the energy and fixed carbon that support marine food webs and carbon reservoirs ${ }^{1}$. However, few phytoplankton are strictly photoautotrophic ${ }^{2}$. Many phytoplankton also utilize dissolved organic matter, taking up particulate detrital organic matter or preying upon other living cells and even harvesting organelles². Mixotrophic lifestyles, in which cells both fix carbon and use exogenously available organic carbon, may enhance fitness when the relative availability of inorganic resources differs from physiological demands ${ }^{3}$. This may occur, for example, where light intensity is low but inorganic nutrients are abundant. Despite the potential importance of mixotrophy to phytoplankton life history, the contribution of heterotrophic carbon assimilation to phytoplankton growth is not well quantified $^{4}$. Simulations suggest that mixotrophy may be a globally significant carbon source for phytoplankton ${ }^{5}$ but this prediction is currently difficult to quantitatively test with empirical data. One reason is that dissolved organic carbon (DOC) in the oceans constitutes an extremely complex mixture of compounds ${ }^{6,7}$, most of which are uncharacterized. This means that uptake measurements using specific organic carbon sources (e.g. glucose, amino acids) ${ }^{8,9}$ do not represent the entirely available DOC pool and may underestimate the actual DOC uptake rates, and hence mixotrophy of major phytoplankton species ${ }^{10}$.

Prochlorococcus are the most abundant phototrophic cells on Earth, actively growing at depths ranging from the ocean surface down to the base of the photic zone $(\sim 160 \mathrm{~m})^{11}$. Across these depths, photosynthetically available radiation (PAR) varies over 3-4 orders of magnitude, a challenge which the diverse Prochlorococcus lineage faces using a variety of adaptations in their photosynthetic apparatus ${ }^{11,12}$. These adaptations have led to the diversification of Prochlorococcus into high-light and low-light adapted clades ${ }^{11,12}$. In addition, Prochlorococcus are mixotrophs, able to uptake dissolved organic compounds such as glucose ${ }^{8}$, pyruvate ${ }^{13}$, amino acids $^{9}$, nucleotides ${ }^{10}$ and perhaps DMSP ${ }^{14,15}$. Yet, to what extent DOC uptake can supplement or replace photosynthetically fixed carbon for respiration and/or growth in this globally-abundant lineage is still unknown ${ }^{10}$. Available evidence suggests that while mixotrophy helps Prochlorococcus survive limited periods of darkness, axenic cells die after 1 week if not exposed to light ${ }^{13,16}$ indicating that light harvesting, and possibly photosynthesis, are likely obligate. assimilation to Prochlorococcus in the oceans. We first use isotopic measurements to quantify 
71 in the Mediterranean Sea. Then we compare observed growth rates from the Pacific Ocean with

72 purely photo-autotrophic growth rates simulated by a laboratory-calibrated photo-physiological

73 model. We also use an individual-based model to illustrate how mixotrophy provides a fitness

74 advantage and deepens the nutricline. Finally, we use time-series observations of vertical profiles

75 of Prochlorococcus ecotypes in subtropical gyres to show that several clades rely extensively on

76 mixotrophic carbon assimilation. Overall, these results suggest that up to a quarter of depth

77 integrated carbon assimilation by Prochlorococcus originates from DOC, with implications for

78 global C cycles, and that mixotrophy is essential to support a significant fraction of

79 Prochlorococcus diversity.

\section{Results and discussion}

82 Carbon and nitrogen uptake in wild samples from the base of the photic zone.

To evaluate the relative contributions of photosynthesis and heterotrophic carbon uptake in a natural Prochlorococcus population from the base of the photic zone, where light may be limiting, we assess the Prochlorococcus population structure and per-cell activity during late summer in the ultra-oligotrophic Eastern Mediterranean $\mathrm{Sea}^{17}$. At the time of sampling, the water column was highly stratified, nutrients were depleted down to around $140 \mathrm{~m}$, and a prominent Deep Chlorophyll Maximum (DCM) was observed at depth of 115 m (Figure 1A). Prochlorococcus were the numerically dominant phytoplankton below the surface (Fig 1B), and could be divided into two populations based on the per-cell fluorescence - a low fluorescence population from the surface to $115 \mathrm{~m}$ and a high fluorescence population from 115-150 m, with an overlap at $115 \mathrm{~m}$ (Figure 1C, D). The shift in the per-cell chlorophyll fluorescence in Prochlorococcus with depth is commonly observed ${ }^{18-20}$, and is usually attributed to a change in the genetic composition of the population, from High-Light adapted cells (HL, low fluorescence) to Low-Light adapted (LL, high fluorescence) ones ${ }^{19}$. However, phenotypic heterogeneity (acclimation) can also contribute to this phenomenon ${ }^{21}$, and indeed amplicon sequencing of the Internal Transcribed Spacer between the $16 \mathrm{~S}$ and $23 \mathrm{~S}$ genes (ITS) ${ }^{21,22}$ revealed a gradual transition from $\mathrm{HL}$ to $\mathrm{LL}$ clades around the DCM, suggesting both genotypic and phenotypic shifts with depth (Figure 1C). The flow cytometry and genetic data are both consistent with previous studies ${ }^{21,23}$, and suggest that the water column had 
101 DCM ( 3-5 $\mu$ mol photons $m^{-2} s^{-1}$ during the afternoon, Figure $1 \mathrm{~A}$ ) is potentially enough under

102 laboratory conditions to support the growth of some LL strains but not sufficient for active growth

103 of most HL strains ${ }^{24}$. Since HL cells comprise $>50 \%$ of the Prochlorococcus population at $115 \mathrm{~m}$

104 and about $25 \%$ at $125 \mathrm{~m}$, this suggests that a significant fraction of the Prochlorococcus cells in

105 these samples are living under conditions where photosynthesis cannot support growth (Figure

106 1C).

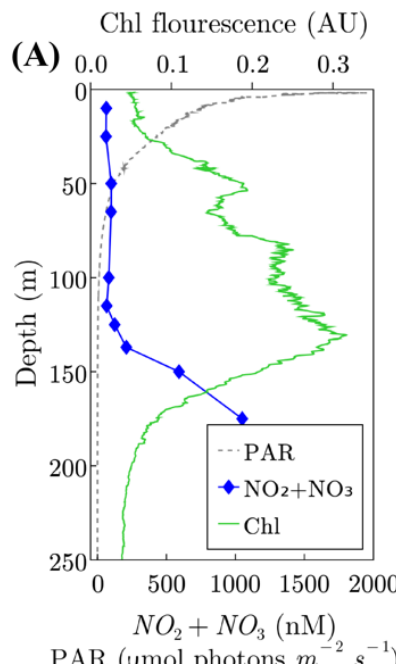

(B)

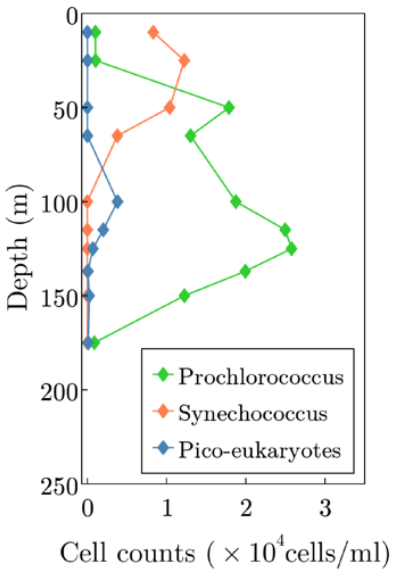

(C)

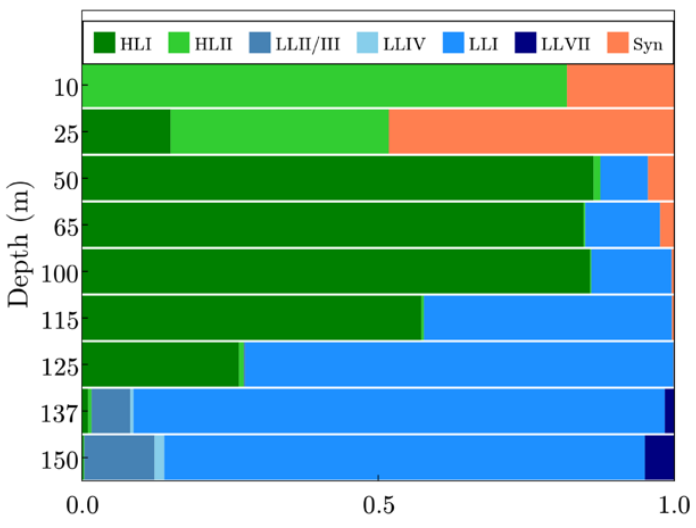

Relative abundance
(D)

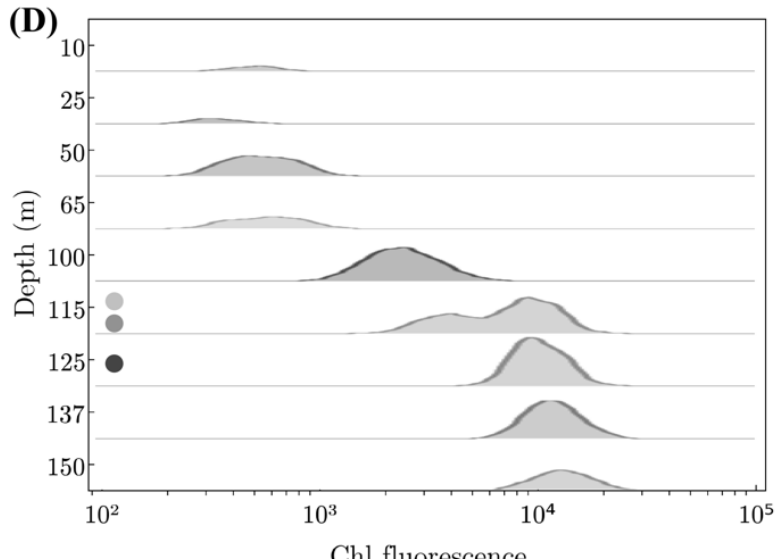

Chl fluorescence

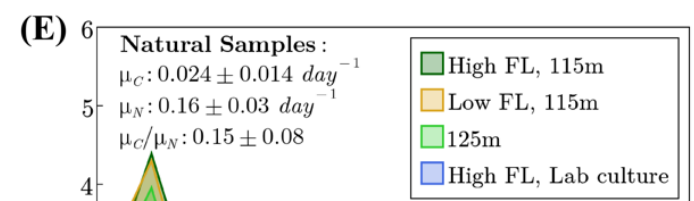

(E)

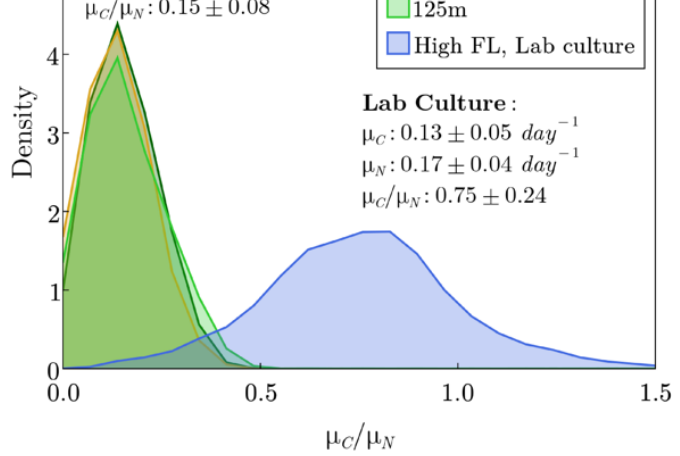

Figure 1: Nutrient uptake of naturally occurring Prochlorococcus populations at the Eastern

Mediterranean Sea. (A) Depth profiles of Photosynthetically Available Radiation (PAR), $\mathrm{NO}_{2}+\mathrm{NO}_{3}$ and Chlorophyll. (B) Phytoplankton cell counts using flow cytometry. (C) Relative abundance of different Prochlorococcus clades across the water column, determined by ITS sequencing. Note the change in Chl fluorescence without a concomitant change in population structure between 60 to $100 \mathrm{~m}$, as well as the presence of LL clades above $115 \mathrm{~m}$ and HL clades at $125 \mathrm{~m}$. (D) Density 
114 plots of Prochlorococcus per-cell chlorophyll fluorescence. Note the double population at $115 \mathrm{~m}$.

115 The circles represent the populations sorted and analyzed by nanoSIMS. (E) Density plots of the 116 ratios of C-specific C uptake rate $\left(\mu_{C}\right)$ to $\mathrm{N}$-specific $\mathrm{N}$ uptake rate $\left(\mu_{N}\right)$ from NanoSIMS analysis of 117 each sorted sub-population from $115 \mathrm{~m}$, the single population from $125 \mathrm{~m}$, and lab cultures. The 118 numbers of cells measured in each population are 45 (LL 115m), 49 (HL 115m), 55 (125m), and 119489 (lab culture).

121 We next measured the uptake of ${ }^{13} \mathrm{C}$-labelled bicarbonate (representing C-fixation through 122 photosynthesis) and of ${ }^{15} \mathrm{~N}$-labeled ammonium (representing nitrogen uptake) in single 123 Prochlorococcus cells from the DCM, using Nanoscale Secondary Ion Mass Spectrometry 124 (NanoSIMS). Essentially all of the Prochlorococcus cells at 115 and $125 \mathrm{~m}$ depth were active 125 (photosynthesized and took up $\mathrm{NH}_{4}$ ). The observation that essentially all of the Prochlorococcus 126 cells in natural samples are active is consistent with a similar study in the North Pacific ${ }^{25}$, and 127 suggests that dead or chlorotic cells observed in laboratory cultures ${ }^{13,26}$ may be relatively rare in 128 nature, at least during midday at the DCM. Nevertheless, the per-cell photosynthesis rates at 129 these depths were not sufficient to support the growth rates indicated by the nitrogen-specific 130 nitrogen uptake rates, even though the uptake experiments were performed when light intensity 131 was maximal (Figure 1E). Previous studies from multiple oceanic regions based on cell cycle 132 analysis and on ${ }^{14} \mathrm{C}$ incorporation into divinyl-chlorophyll indicate that Prochlorococcus cells at 133 depths of 100-150 m replicate every 4-7 days (a growth rate of 0.14-0.25 day $\left.{ }^{-1}\right)^{27-30}$. However, 134 the observed $\mathrm{C}$-specific $\mathrm{C}$ uptake rate $\left(\mu_{C}\right)$ was only $\sim 0.024$ day $^{-1}$, too low to support these expected growth rates, while the observed $\mathrm{N}$-specific $\mathrm{N}$ uptake rate $\left(\mu_{N}\right)$ was $\sim 0.16$ day $^{-1}$ 137 than normal cells which are expected to be $\sim 1\left(\mu_{C} \approx \mu_{N}\right)$. Indeed, $\mu_{C} / \mu_{N}$ in lab cultured 138 Prochlorococcus was $\sim 0.75$ (Figure $1 \mathrm{E}$ ). Taken together, these quantitative observations suggest 139 that $>80 \%$ of the $C$ required for the expected growth rate of these Prochlorococcus cells at the DCM must come from non-photosynthetic sources. 
144 in the deeper reaches of the photic zone is of organic origin. By comparing measured profiles of growth rates and modeling photosynthetic carbon fixation from sites in the Pacific, we ask if this is consistent in other regions and infers the water column integrated contribution of heterotrophy. Vaulot et al. ${ }^{31}$ and Liu et al. ${ }^{32}$ reported vertical profiles of Prochlorococcus division rates based on cell-cycle analysis in the Equatorial Pacific (EqPac, $0^{\circ} \mathrm{N}, 140^{\circ} \mathrm{W}$ ) and North Pacific Subtropical Gyre (HOT, $22^{\circ} 45^{\prime} \mathrm{N}, 158^{\circ} \mathrm{W}$; Station ALOHA), respectively. These data were obtained in the context of an extensive biogeochemical survey (JGOFS EqPac) ${ }^{33}$ and time-series station $151(\mathrm{HOT})^{34}$ and are associated with rich contextual data sets including observations of cell counts, 152 photon fluxes and nutrient concentrations (Figure 2A, C). Calibrated by observed, noon-time PAR 153 profiles, we simulated the daily cycle of photosynthesis and the vertical profiles of 154 Prochlorococcus' carbon-specific, net photosynthesis rate $\left(\right.$ day $\left.^{-1}\right)$. We simulated both HL and LL ecotypes, using laboratory calibrations of the photosynthesis-irradiance relationship from Moore and Chisholm ${ }^{24}$. Similarly, using allometric scaling for fixed-nitrogen, phosphate and dissolved iron uptake rates ${ }^{35,36}$, along with observed environmental concentrations, we evaluated the nutrientspecific uptake rates $\left(d_{a y}{ }^{-1}\right)$. Full details are presented in Materials and Methods. The estimated, purely autotrophic growth rates were determined by the most limiting resource at each depth (Figure 2B, D). Light and carbon fixation strongly limited the simulated autotrophic growth in the deeper region of the photic layer, while fixed nitrogen (HOT), iron (EqPac) and carbon fixation, due to photo-inhibition, were important near the surface (Figure 2). While the observed growth rates at the surface were mostly within the ranges predicted from the photophysiological parameters of $\mathrm{HL}$ and $\mathrm{LL}$ strains (blue shade in Figure $2 \mathrm{~B}$ and $\mathrm{D}$ ), the model failed to resolve the observed growth rates below $\sim 75-100 \mathrm{~m}$ at both stations. Rather, the model unequivocally suggests that photosynthesis alone cannot account for the observed division rates at depth. We interpret the differences between the modeled autotrophic and observed actual growth rates at depth (red shading) to infer the minimal rate of organic carbon assimilation of Prochlorococcus. The two stations represent very different physical and biogeochemical regimes, yet show similar 170 qualitative structure. Mixotrophy appears to become significant at different depths at the two 171 stations (95 $\mathrm{m}$ at HOT and $60 \mathrm{~m}$ at EqPac) but at similar level of PAR ( $15 \mu \mathrm{mol}$ photons $\mathrm{m}^{-2} \mathrm{~s}^{-1}$, $172 \sim 5 \%$ of surface PAR). Using observed cell densities ${ }^{31,32}$ and assumed cellular carbon quotas ${ }^{37}$ we 173 estimated the vertically integrated autotrophic net primary production for Prochlorococcus to be $174 \sim 0.35 \mathrm{gC} \mathrm{m}^{-2} \mathrm{day}^{-1}$ at HOT and $\sim 0.20 \mathrm{gC} \mathrm{m}^{-2} \mathrm{day}^{-1}$ at EqPac, with vertically integrated 
175 heterotrophic contributions (based on the red shading in Figures 2B and D) of $\sim 0.075$ $176 \mathrm{gC} \mathrm{m} \mathrm{m}^{-2} \mathrm{day}^{-1}$ at HOT and $0.069 \mathrm{gC} \mathrm{m}^{-2} \mathrm{day}^{-1}$ at EqPac. In other words, assimilation of 177 organic carbon is inferred to support $18 \%$ of total Prochlorococcus biomass production at HOT 178 and $\sim 25 \%$ at EqPac. Furthermore, organic carbon uptake contributes $\sim 80 \%$ at HOT and $54 \%$ at $179 \mathrm{EqPac}$ of the total production below the depth where the contribution of mixotrophy is greater 180 than photosynthesis, broadly consistent with the isotopic inference from the deep photic zone in the Mediterranean. We note that this model does not take into account exudation of organic carbon by Prochlorococcus which is not well constrained experimentally and would likely reduce the inferred growth rates at the surface ${ }^{38-41}$. Indeed, mixotrophy (uptake of glucose and amino acids) has been observed in surface Prochlorococcus ${ }^{9,10}$, suggesting that our estimate provides a lower bound of the contribution of mixotrophy to integrated Prochlorococcus production.

(A) PAR ( $\mu$ mol photons $\left.m^{-2} s^{-1}\right)$

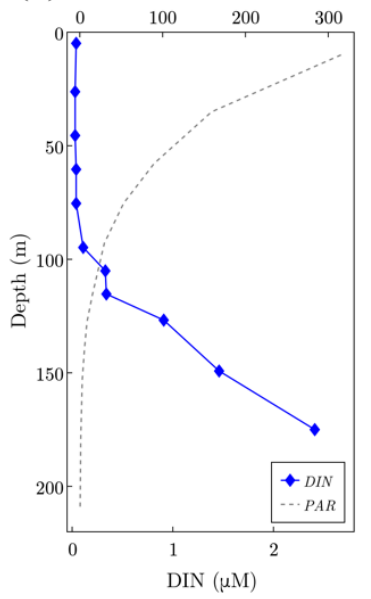

(B)

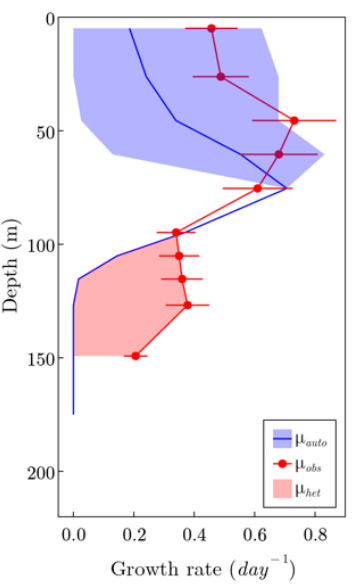

(C) PAR ( $\mu$ mol photons $\left.m^{-2} s^{-1}\right)$

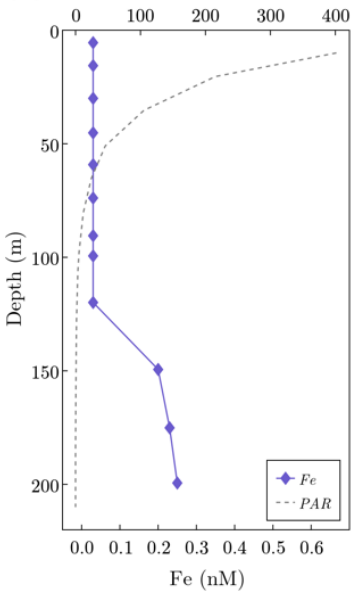

(D)

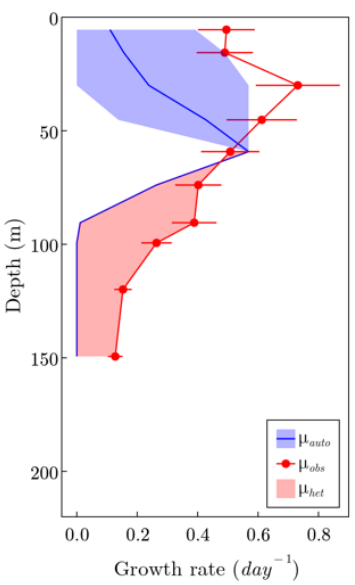

Figure 2. Simulated growth rates at HOT and EqPac. (A), (C) Observations of PAR and dissolved inorganic nitrogen (DIN) at the Hawaii Ocean Time-series (HOT, panel A) and of PAR and dissolved iron (Fe) at the equatorial Pacific (EqPac, panel C). (B), (D) Simulated autotrophic growth rate (blue line) and observed growth rates (red line with dots, data from ${ }^{42}$ ) at HOT (B) and EqPac (D). The blue shade represents the difference between simulated HL and LL ecotypes, and the red shade represents the inferred heterotrophic growth rate. A $19 \%$ error of observed growth rate is included both at HOT and EqPac according to Vaulot et al. ${ }^{31}$. 
196 To investigate the implications of mixotrophy on biogeochemical dynamics, we employed an

197 individual-based modeling approach (see Method for details), simulating trajectories of individual

198 Prochlorococcus cells (or super-agents representing many cells) through light and nutrient

199 environments in a two-dimensional, highly resolved turbulent fluid flow (see supporting movie).

200 Inorganic nutrients and a DOC-like tracer are represented by density-based equations. Briefly,

201 individuals fix carbon by photosynthesis and take up inorganic nitrogen and phosphorus. Two

202 idealized types of individuals are simulated separately, one with a strict photo-autotrophic

203 lifestyle and the other which is mixotrophic and able to assimilate carbon from the DOC-like

204 substance. The mixotrophic individual cannot live strictly heterotrophically, as suggested by Coe

205 et al. $^{13}$, which we parameterize as requiring $1 \%$ of the incorporated $\mathrm{C}$ to come from

206 photosynthesis. In Figure 3A we illustrate horizontally-averaged profiles of cell density from the

207 purely autotrophic and mixotrophic simulations, illustrating how mixotrophy supports a

208 population of Prochlorococcus below $\sim 75 \mathrm{~m}$. The simulated daily division rate of $\sim 0.2$ day $^{-1}$ at

209 depth (Figure 3B) is consistent with the published cell-cycle profiles from the subtropical and the

210 Equatorial Pacific ${ }^{31,32}$ and is a bit higher than the aforementioned inferred division rate in the

211 Mediterranean based on $\mathrm{NH}_{4}$ uptake. Mixotrophs and autotrophs share the same division rate

$212\left(\sim 0.3 \mathrm{day}^{-1}\right)$ in the mixed layer (surface $50 \mathrm{~m}$ ) where the inorganic nutrient is the limiting factor

213 in the simulations. The autotrophs then reach a maximum daily division rate of $\sim 0.5 \mathrm{day}^{-1}$ at 60

$214 \mathrm{~m}$ depth where the transition of $\mathrm{N}$ to $\mathrm{C}$ limitation happens, and then decrease rapidly to zero at

$21590 \mathrm{~m}$ depth due to light limitation. In contrast, the mixotrophs have a deeper maximum growth

216 rate of $0.5 \mathrm{day}^{-1}$ at $80 \mathrm{~m}$ depth where the transition of $\mathrm{N}$ to $\mathrm{C}$ limitation occurs and gradually

217 decrease to $\sim 0.2$ day $^{-1}$ at $125 \mathrm{~m}$ depth (Figure 3B). The deeper maximum division depth of the

218 mixotrophs and their ability to maintain a population at depths where photosynthesis is not

219 sufficient are supported by the DOC utilization, which is presented as a black line in Figure 3B. In

220 the mixotrophic simulation, the contribution of DOC uptake to the vertically integrated total

221 production is 12\%; 43\% when light becomes the limiting factor, below the red stripe in Figure

222 3B. The contribution of DOC and the maximal depth at which Prochlorococcus can grow are

223 broadly consistent with the division rate profile model and are sensitive to parameter values

224 which control the nutritional value of the DOC-like substance (and which cannot be a priori

225 constrained by empirical data at this point; see Materials and Methods). Notably, the horizontal

226 stripes in Figure 3B indicate the depth at which limitation shifted from nutrients to $C$ in the two 
(A) PAR ( $\mu$ mol photons $\left.m^{-2} s^{-1}\right)$

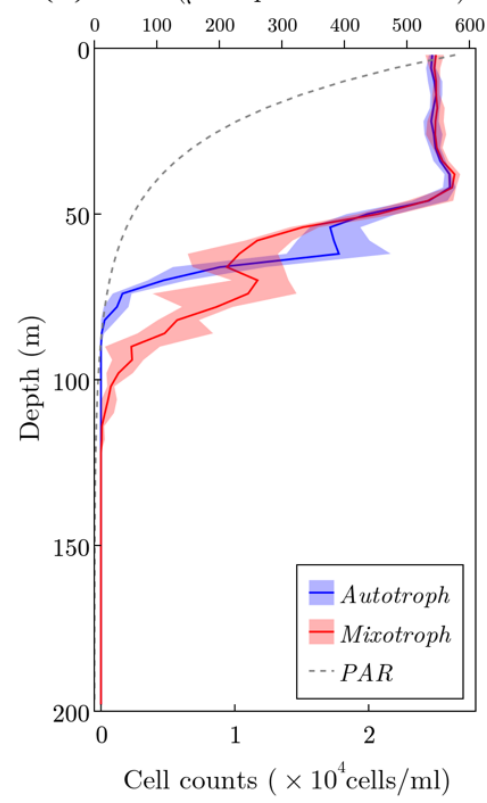

(B) Contribution of DOC uptake (\%)

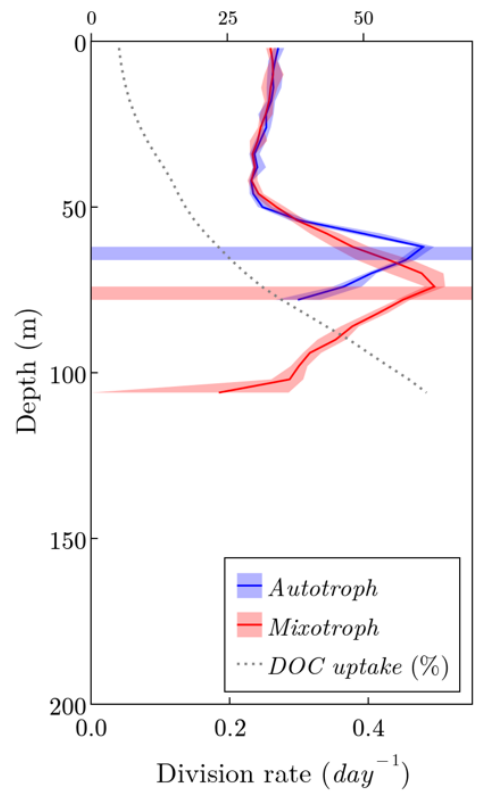

(C)

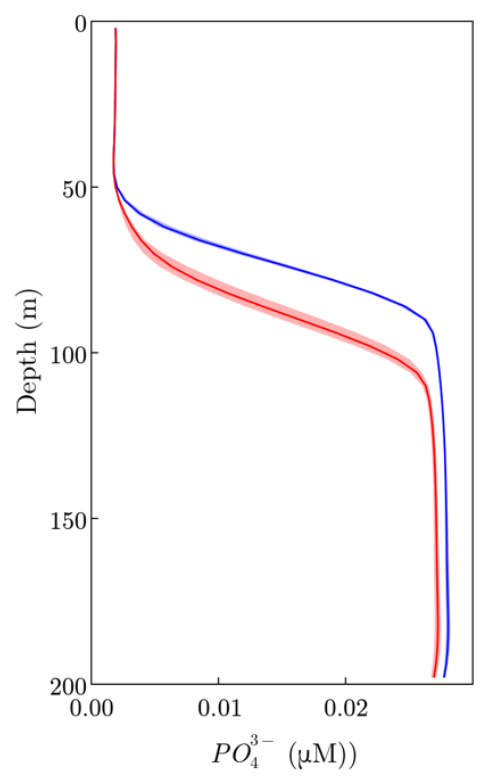

230 Figure 3. Vertical profiles of simulated autotrophs and mixotrophs in the individual-based

231 model. The red and blue shades in all panels indicate the differences between an ensemble of 10 232 model runs. (A) Vertical profiles of cell density of simulated autotrophs (blue) and mixotrophs 233 (red). The vertical profile of PAR is represented as the gray dashed line. (B) Vertical profiles of cell 234 division rate of autotrophs (blue) and mixotrophs (red). The blue and red stripes indicate the 235 transition point from nutrient limitation to carbon limitation of phytoplankton growth. The black 236 dotted line represents the contributions of DOC uptake to total carbon acquisition in mixotrophs.

237 (C) Vertical profiles of phosphate in simulations of autotroph (blue) and mixotroph (red). Interpretation of vertical distributions of Prochlorococcus ecotype at time-series stations.

240 To what extent does mixotrophy supports natural, genetically-diverse, populations of 241 Prochlorococcus? To answer this question, we calculated the fraction of the Prochlorococcus cells 242 and of individual ecotypes living below the depth where they can be supported by photosynthesis 243 alone over a 5-year time series in the north Atlantic and Pacific gyres (Hawaii and Bermuda time 244 series study sites, respectively ${ }^{23}$ ). We consider only the time of the year when the water column 
245 is stratified (white regions in Figure 4), defined here as a mixed layer depth that is shallower than

246 the photic depth (light intensity is $>10 \mu$ mol photons $m^{-2} s^{-1}$ for high-light strains or $>2.8$

$247 \mu$ mol photons $m^{-2} s^{-1}$ for low-light strains, experimentally-determined minimal light

248 requirement for active growth of high-light and low-light adapted strains during a 14:10 day-night

249 cycle $\left.{ }^{24}\right)$. This is because at other times cells below the photic depth but still within the upper mixed

250 layer could be transferred closer to the surface and therefore receive increased light. An average

251 of $\sim 8-10 \%$ of the Prochlorococcus cells during these stratified periods are likely to be light-starved

252 (Figure 4 A, B), including the vast majority of LL adapted ecotypes (Figure 4C, D).
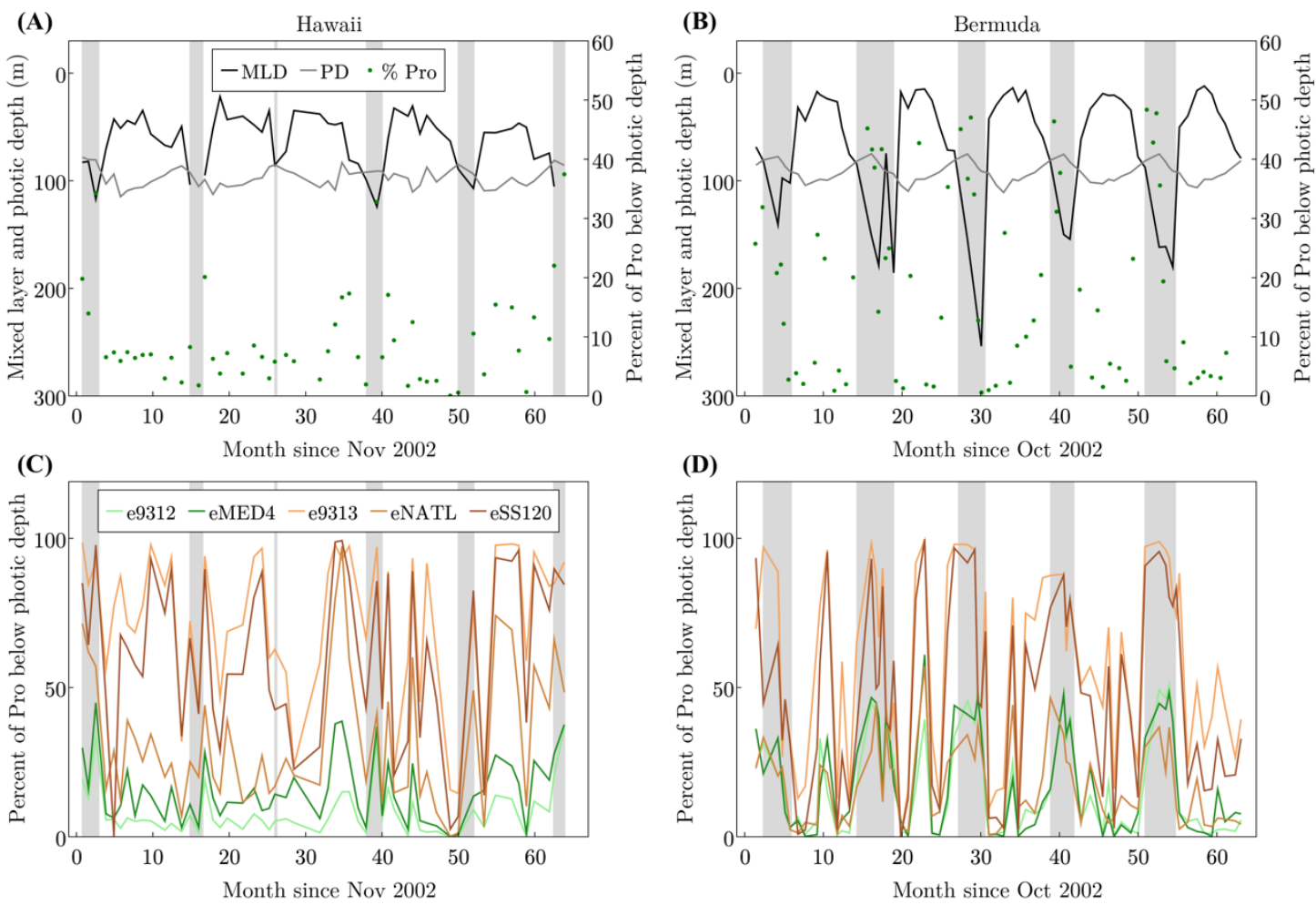

253

254 Figure 4: Estimating the number of Prochlorococcus cells and of specific ecotypes found below

255 their photic depth at Hawaii and Bermuda. (A), (B) The percent of total Prochlorococcus cells

256 (Pro) found below their photic zone at Hawaii (A) and Bermuda (B), defined as the integrated

257 illumination level supporting the growth of representative strains in laboratory cultures ${ }^{24}$ (grey

258 line shows this depth for HL strains). The black line shows the mixed layer depth (MLD), the grey

259 line shows the photic depth (PD), the green dots represent the percentages of Prochlorococcus below photic depth, the grey areas are non-stratified conditions where cells may be mixed from 
261 depth to the surface. (C), (D) The percentage of each Prochlorococcus ecotype below its photic

262 depth. The data are taken from Malmstrom et al. ${ }^{23}$.

\section{Conclusions}

265 We have presented several lines of evidence illustrating the importance of mixotrophic carbon 266 assimilation by Prochlorococcus. The uptake of isotopically labelled nitrogen uptake in samples 267 from the Mediterranean Sea indicate doubling times at the DCM of about a week, consistent with 268 cell-cycle based observations from the Equatorial and Subtropical Pacific ${ }^{27,29-32}$. The associated 269 uptake of labelled carbon suggests that this growth rate is only viable if more than three-quarters 270 of assimilated carbon is sourced from organic matter. Using a laboratory-calibrated model of 271 carbon-specific photosynthesis rates and local environmental data, we compared carbon-limited 272 growth rates with observed cell-cycle observations at the Pacific locations. We estimated that $27318-25 \%$ of depth integrated, net carbon assimilation by Prochlorococcus is heterotrophic at those 274 sites, with as much as $80 \%$ heterotrophic carbon supply at the DCM. We note that while this shifts 275 perception of the photo-autotrophic nature of primary producers, products such as remote276 sensing based estimates of global-scale primary production are typically calibrated with data from 277 isotopically labeled inorganic carbon studies and hence, other sources of error notwithstanding, 278 are appropriately estimating photosynthesis and not growth rates. We explored the wider 279 consequences of the phenomenon in simulations with an individual-based model that resolves a 280 DOC-like substance. These simulations suggest that such extensive mixotrophy in the deeper 281 photic layer will significantly deepen the nutricline. This is significant for carbon cycle simulations, 282 most of which do not currently resolve mixotrophy and may predict, or inappropriately tune, a 283 too-shallow nutricline. Finally, investigation of the ecotypic, vertical biogeography in the 284 subtropical North Pacific and North Atlantic ${ }^{23}$ indicates that low-light adapted Prochlorococcus 285 spend $50-100 \%$ of their time, depending on season, below the deepest horizon for photo286 autotrophically viable maintenance of the population. We propose that reliance on mixotrophy, 287 rather than on photosynthesis, underpins the ecological success of a large fraction of the global 288 Prochlorococcus population and its collective genetic diversity. 
Mediterranean seawater was collected during August 2017 (station N1200, 32.45 $\mathrm{N}, 34.37^{\circ} \mathrm{E}$ )

from 11 depths by Niskin bottles and divided into triplicate $250 \mathrm{ml}$ polycarbonate bottles. Two bottles from each depth were labeled with $1 \mathrm{mM}$ Sodium bicarbonate- ${ }^{13} \mathrm{C}$ and $1 \mathrm{mM}$ Ammonium-

${ }^{15} \mathrm{~N}$ chloride (Sigma-Aldrich, USA) and all 3 bottles ( 2 labelled and 1 control) were incubated at the original depth and station at sea for 3.5 hours around mid-day. The short incubation time was chosen to minimize isotope dilution and potential recycling and transfer of ${ }^{13} \mathrm{C}$ and ${ }^{15} \mathrm{~N}$ between community members ${ }^{25}$. After incubation, bottles were brought back on board and the incubations were stopped by fixing with 2 X EM grade glutaraldehyde (2.5\% final concentration) and stored at $4{ }^{\circ} \mathrm{C}$ until sorting analysis. Cell sorting, NanoSIMS analyses and the calculation of uptake rates were performed as described in Roth-Rosenberg et al. ${ }^{26}$.

DNA collection and extraction from seawater

Samples for DNA were collected on $0.22 \mu \mathrm{m}$ Sterivex filters (Millipore). Excess water was removed using a syringe, $1 \mathrm{ml}$ Lysis buffer (40 mM EDTA, $50 \mathrm{mM}$ Tris $\mathrm{pH}$ 8.3, $0.75 \mathrm{M}$ sucrose) was added and both ends of the filter were closed with parafilm. Samples were kept at $-80^{\circ} \mathrm{C}$ until extraction. DNA was extracted by using a semi-automated protocol including manual chemical cell lysis before the automated steps. The manual protocol began with thawing the samples, then the storage buffer was removed using a syringe and $170 \mu$ lysis buffer added to the filters. $30 \mu$ of Lysozyme $(20 \mathrm{mg} / \mathrm{ml})$ were added to the filters and incubated at $37^{\circ} \mathrm{C}$ for $30 \mathrm{~min}$. After incubation,

$31120 \mu$ l proteinase $\mathrm{K}$ and $200 \mu \mathrm{l}$ buffer $\mathrm{AL}$ were added to the tube for 1 hour at $56^{\circ} \mathrm{C}$ (with agitation).

312 The supernatant was transferred to a new tube and DNA was extracted using the QIAcube 313 automated system and QIAamp DNA Mini Protocol: DNA Purification from Blood or Body Fluids 314 (Spin Protocol, starting from step 6, at the BioRap unit, Faculty of Medicine, Technion). All DNA 315 samples were eluted in $100 \mu$ I DNA free distilled-water.

318 PCR amplification of the ITS was carried out with specific primers for Prochlorococcus 319 CS1_16S_1247F (5'-ACACTGACGACATGGTTCTACACGTACTACAATGCTACGG) and Cs2_ITS_Ar (5'TACGGTAGCAGAGACTTGGTCTGGACCTCACCCTTATCAGGG) ${ }^{21,22}$. The first PCR was performed in 
322 (Bioline) and $0.5 \mu \mathrm{l}$ of $10 \mu \mathrm{M}$ of each primer. The amplification conditions comprised steps at $95^{\circ} \mathrm{C}$

323 for $5 \mathrm{~min}, 28 / 25$ (16S/ITS) cycles at $95^{\circ} \mathrm{C}$ for $30 \mathrm{sec}, 50^{\circ} \mathrm{C}$ for $30 \mathrm{sec}$ and $72^{\circ} \mathrm{C}$ for $1 \mathrm{~min}$ followed

324 by one step of $5 \mathrm{~min}$ at $72^{\circ} \mathrm{C}$. All PCR products were validated on a $1 \%$ agarose gel and triplicates

325 were pooled. Subsequently, a second PCR amplification was performed to prepare libraries.

326 These were pooled and after a quality control sequenced (2×250 paired-end reads) using an

327 Illumina MiSeq sequencer. Library preparation and pooling were performed at the DNA Services

328 (DNAS) facility, Research Resources Center (RRC), University of Illinois at Chicago (UIC). MiSeq

329 sequencing was performed at the W.M. Keck Center for Comparative and Functional Genomics at

330 the University of Illinois at Urbana-Champaign (UIUC).

ITS Sequence processing

333 Paired-end reads were analyzed using the Dada2 pipeline ${ }^{43}$. The quality of the sequences per 334 sample was examined using the Dada2 'plotQualityProfile' command. Quality filtering was 335 performed using the Dada2 'filterAndTrim' command with parameters for quality filtering 336 truncLen $=c(290,260)$, $\max N=0, \max E E=c(2,2)$, truncQ $=2$, rm.phix $=T R U E$, trimLeft $=c(20,20)$.

337 Following error estimation and dereplication, the Dada2 algorithm was used to correct sequences.

338 Merging of the forward and reverse reads was done with minimum overlap of $4 \mathrm{bp}$. Detection and 339 removal of suspected chimeras was done with command 'removeBimeraDenovo'. In total, 340388,417 sequences in 484 amplicon sequence variants (ASVs) were counted. The ASVs were 341 aligned in MEGA6 ${ }^{44}$ and the first 295 nucleotides, corresponding to the 165 gene, were trimmed.

342 The ITS sequences were then classified using BLAST against a custom database of ITS sequences 343 from cultured Prochlorococcus and Synechococcus strains as well as from uncultured HL and LL 344 clades.

347 PlanktonIndividuals.jl (v0.1.9) was used to run the individual-based simulations. A full 348 documentation is available at https://juliaocean.github.io/PlanktonIndividuals.jl/dev/. Briefly, the 349 cells fix inorganic carbon through photosynthesis and nitrogen, phosphorus and DOC from the 350 water column and grow until division or grazing. Cell division is modeled as a probabilistic function 351 of cell size. Grazing is represented by a quadratic probabilistic function of cell population. Cells 352 consume nutrient resources which are represented as Eulerian, density-based tracers. We set up 
two separate simulations, each of them either has a population of an obligate photo-autotroph or a mixotroph which also consumes DOC. The initial conditions and parameters are the same for the two simulations except the ability of mixotrophy. The simulations were run with a time step of 1 minute for 360 simulated days to achieve a steady state. We run the two simulations for multiple times in order to get the range of the stochastic processes. The code of this configuration is available at https://github.com/zhenwu0728/Prochlorococcus Mixotrophy.

Evaluation of autotrophic growth rates.

361 We evaluated the carbon-specific, daily-averaged carbon fixation rate, $\mathbb{P}$ as a function of light intensity $(I, \mu E)$ as follows:

$$
\mathbb{P}=\frac{1}{\Delta t} \int_{0}^{\Delta t} \frac{q_{C h l}}{q_{C}} P_{S}^{C h l}\left(1-e^{-\alpha_{C h l} I / P_{S}^{C h l}}\right) e^{-\beta_{C h l} I / P_{S}^{C h l}} d t
$$

Here, following Platt et al. ${ }^{45}: P_{S}^{C h l}$ is an empirically constrained coefficient representing the Chlorophyll-a specific carbon fixation rate $\left(\mathrm{mol} \mathrm{C} \cdot(\mathrm{mol} \mathrm{Chl})^{-1} \cdot s^{-1}\right)$ and $\frac{q_{C h l}}{q_{C}}$ is the molar

366 Chlorophyll-a to carbon ratio. $\alpha_{C h l}$ and $\beta_{C h l}$ are empirically determined coefficients representing 367 the initial slope of the photosynthesis-light relationship and photo-inhibition effects at high photon fluxes, respectively. Here we impose empirically determined values for $\alpha_{C h l}$ and $\beta_{C h l}$ and $P_{S}^{C h l}$ from the published study of Moore and Chisholm ${ }^{24}$. To find the maximum estimate for Prochlorococcus photosynthesis at different light intensities we use photo-physiological

$372 S^{-1}$ and a Low-Light adapted ecotype (MIT9211), acclimated $9 \mu E . \Delta \mathrm{t}=24$ hours. $I$ is the hourly 373 PAR, estimated by scaling the observed noon value at each depth with a diurnal variation 374 evaluated from astronomical formulae based on geographic location and time of year ${ }^{33,34}$. The 375 Chlorophyll to Carbon ratio, $\frac{q_{C h l}}{q_{C}}$, is estimated as a function of growth rate and light intensity using 376 the model of Inomura et al. ${ }^{46}$ which was calibrated by laboratory data from Healey ${ }^{47}$.

377 The Chlorophyll to carbon ratio, $\frac{q_{C h l}}{q_{C}}$, can be modeled as a function of growth rate and light 378 intensity ${ }^{46,48}$. Here we use the Inomura ${ }^{46}$ model (equation 17 therein) where parameters were 379 calibrated with laboratory data from Healey ${ }^{47}$. An initial guess of the growth rate and the 
380 empirically informed light intensity are used to estimate $\frac{q_{C h l}}{q_{C}}$, which is then used to evaluate the 381 light-limited, photoautotrophic growth rate

$$
\mathbb{V}_{C}^{\text {auto }}=\frac{P}{q_{C}}
$$

from which the Chlorophyll to carbon ratio is again updated. The light-limited growth rate is used

384 to re-evaluate the Chlorophyll to carbon ratio. Repeating this sequence until the values converge, $\mathbb{V}_{C}^{\text {auto }}$ and $\frac{q_{C h l}}{q_{C}}$ are solved iteratively.

The nitrogen-specific uptake rate of fixed nitrogen $\left(d a y^{-1}\right)$ is modeled as

$$
\mathbb{V}_{\mathrm{N}}=\mathbb{V}_{\mathrm{N}}^{\max } \frac{1}{\mathrm{Q}_{\mathrm{N}}} \frac{\mathrm{N}}{\mathrm{N}+\mathrm{K}_{\mathrm{N}}}
$$

where values of the maximum uptake rate, $\mathbb{V}_{N}^{\max }$ and half-saturation, $K_{N}$, are determined from fmol $N$ cell $\left.^{-1}\right)$.

391 The P-limited growth rate, or the phosphorus-specific uptake rate of phosphate $\left(\right.$ day $\left.^{-1}\right)$, is modeled as

$$
\mathbb{V}_{P}=\mathbb{V}_{P}^{\max } \frac{1}{Q_{P}} \frac{P O_{4}^{3-}}{P O_{4}^{3-}+K_{P}}
$$

394 where values of the maximum uptake rate, $\mathbb{V}_{P}^{\max }$ and half-saturation, $K_{P}$, are determined from 395 empirical allometric scalings ${ }^{35}$, along with a nitrogen cell quota $Q_{P}$ from Bertilsson et al. ${ }^{37}(0.048$ 396 fmol P cell $\left.^{-1}\right)$.

Iron uptake is modeled as a linear function of cell surface area $(S A)$, with rate constant $\left(k_{F e}^{S A}\right)$

399 following Shaked et al. ${ }^{36}$.

$$
\mathbb{V}_{F e}=k_{F e}^{S A} \cdot S A \frac{1}{Q_{F e}} F e
$$

401 The potential light-, nitrogen-, phosphorus- and iron-limited growth rates $\left(\mathbb{V}_{C}, \mathbb{V}_{N}, \mathbb{V}_{P}, \mathbb{V}_{F e}\right)$ were evaluated at each depth in the water column and the minimum is the local modeled photo- 
autotrophic growth rate estimate, absent of mixotrophy (blue lines, Figure 2B, D). The the model

404 is available at https://github.com/zhenwu0728/Prochlorococcus Mixotrophy.

406 A significant premise of this study is that heterotrophy is providing for the shortfall in carbon 407 under very low light conditions, but not nitrogen. It is known that Prochlorococcus can assimilate 408 amino acids $^{9}$ and therefore the stoichiometry of the heterotrophic contribution might alter the 409 interpretations. However, it is also known that Prochlorococcus can exude amino acids ${ }^{38}$ which 410 might cancel out the effects on the stoichiometry of Prochlorococcus.

411 For the estimates of photo-trophic growth rate from local environmental conditions (Figure 2) we 412 employed photo-physiological parameters from laboratory cultures of Prochlorococcus ${ }^{24}$. For the 413 purposes of this study, we have assumed that the photosynthetic rates predicted are Net Primary 414 Production which means that autotrophic respiration has been accounted for in the 415 measurement. However, the incubations in that study were of relatively short timescale (45 $\mathrm{min}$ ), 416 which might suggest they are perhaps more representative of Gross Primary Production. If this is 417 the case, our estimates of photo-autotrophic would be even lower after accounting for 418 autotrophic respiration, and thus would demand a higher contribution from heterotrophic carbon 419 uptake. In this regard, our estimates might be considered a lower bound for organic carbon 420 assimilation.

\section{Acknowledgements}

423 We thank the captain and crew of the R/V Mediterranean Explorer and Tom Reich, for help during 424 the work at sea, Mike Krom and Anat Tsemel for the nutrient analyses, Maya Ofek-Lalzar for 425 assistance with the bioinformatics analysis, Annett Grüttmüller for NanoSIMS routine operation, 426 Ioannis Tsakalakis for help with hourly PAR estimation, and John Casey for the discussion about C 427 uptakes rates. This study was supported by grant RGP0020/2016 from the Human Frontiers 428 Science Program (to MV, HPG and DS) and by grant number 1635070/2016532 from the NSF-BSF 429 program in Oceanography (NSFOCE-BSF, to DS). The NanoSIMS at the Leibnitz-Institute for Baltic 430 Sea research in Warnemuende (IOW) was funded by the German Federal Ministry of Education 431 and Research (BMBF), grant identifier 03F0626A. MJF and WZ are grateful for support from the 432 Simons Foundation through the Simons Collaboration on Ocean Processes and Ecology (SCOPE 
329108 to MJF) and the Simons Collaboration for Computational BIOgeochemical Modeling of

434 marine EcosystemS (CBIOMES 549931 to MJF).

436 Author contributions

437 DA, DRR, TLK, AV, MV and DS designed experiments, DRR, DA, TLK, LZ and DS performed 438 experiments and field analyses, DRR, DA, TLK, AV, and FE performed NanoSIMS analyses, DA, DRR,

439 TLK, AV, LZ, FE, HPG, MV and DS analyzed experimental results. WZ, MJF, OW and DS designed

440 and executed the growth rate simulations. WZ designed and executed the individual-based model

441 simulations. WZ, DA, DRR, TLK, MJF and DS wrote the manuscript with contributions from all

442 authors.

\section{Competing interests}

445 The authors declare no competing interests.

\section{References}

$448 \quad$ 1. Falkowski, P. G. The role of phytoplankton photosynthesis in global biogeochemical cycles. Photosynthesis Research 1994 39:3 39, 235-258 (1994).

$450 \quad 2 . \quad$ Stoecker, D. K., Hansen, P. J., Caron, D. A. \& Mitra, A. Mixotrophy in the Marine Plankton.

3. Hartmann, M. et al. Mixotrophic basis of Atlantic oligotrophic ecosystems. Proceedings of

4. Zubkov, M. v. \& Tarran, G. A. High bacterivory by the smallest phytoplankton in the North

5. Ward, B. A. \& Follows, M. J. Marine mixotrophy increases trophic transfer efficiency,

6. Repeta, D. J. Unifying chemical and biological perspectives of carbon accumulation in the Sciences 113, 2958-2963 (2016). environment. Proceedings of the National Academy of Sciences of the United States of America vol. 118 (2021).

7. Zakem, E. J., Cael, B. B. \& Levine, N. M. A unified theory for organic matter accumulation. Proceedings of the National Academy of Sciences of the United States of America 118, (2021).

8. Munoz-Marin, M. d. C. et al. Prochlorococcus can use the Pro1404 transporter to take up glucose at nanomolar concentrations in the Atlantic Ocean. Proceedings of the National Academy of Sciences 110, 8597-8602 (2013). 
9. Zubkov, M. v., Tarran, G. A. \& Fuchs, B. M. Depth related amino acid uptake by Prochlorococcus cyanobacteria in the Southern Atlantic tropical gyre. FEMS Microbiology Ecology 50, 153-161 (2004).

10. Muñoz-Marín, M. C. et al. Mixotrophy in marine picocyanobacteria: use of organic compounds by Prochlorococcus and Synechococcus. The ISME Journal 14, 1065-1073 (2020).

11. Biller, S. J., Berube, P. M., Lindell, D. \& Chisholm, S. W. Prochlorococcus: the structure

12. Rocap, G. et al. Genome divergence in two Prochlorococcus ecotypes reflects oceanic niche differentiation. Nature 2003 424:6952 424, 1042-1047 (2003).

13. Coe, A. et al. Survival of Prochlorococcus in extended darkness. Limnology and Oceanography 61, 1375-1388 (2016).

14. Vila-Costa, M. et al. Dimethylsulfoniopropionate Uptake by Marine Phytoplankton. Science 314, 652-654 (2006).

15. Becker, J. W., Hogle, S. L., Rosendo, K. \& Chisholm, S. W. Co-culture and biogeography of Prochlorococcus and SAR11. The ISME Journal 13, 1506-1519 (2019).

16. Coe, A. et al. Coping with darkness: The adaptive response of marine picocyanobacteria to repeated light energy deprivation. Limnology and Oceanography 66, 3300-3312 (2021).

18. Campbell, L. \& Vaulot, D. Photosynthetic picoplankton community structure in the

17. Reich, T. et al. Seasonal dynamics of phytoplankton and bacterioplankton at the ultraoligotrophic southeastern Mediterranean Sea. bioRxiv 2021.03.24.436734 (2021) doi:10.1101/2021.03.24.436734. subtropical North Pacific Ocean near Hawaii (station ALOHA). Deep Sea Research Part I: Oceanographic Research Papers 40, 2043-2060 (1993).

19. Moore, L. R., Rocap, G. \& Chisholm, S. W. Physiology and molecular phylogeny of coexisting Prochlorococcus ecotypes. Nature 393, 464-467 (1998).

20. van den Engh, G. J. et al. Dynamics of Prochlorococcus and Synechococcus at Station ALOHA Revealed through Flow Cytometry and High-Resolution Vertical Sampling. Frontiers in Marine Science 4, 359 (2017).

21. Thompson, A. W. et al. Dynamics of Prochlorococcus Diversity and Photoacclimation During Short-Term Shifts in Water Column Stratification at Station ALOHA. Frontiers in Marine Science 5, 488 (2018).

22. Ahlgren, N. A., Perelman, J. N., Yeh, Y. \& Fuhrman, J. A. Multi-year dynamics of fine-scale marine cyanobacterial populations are more strongly explained by phage interactions than abiotic, bottom-up factors. Environmental Microbiology 21, 2948-2963 (2019). 
504 23. Malmstrom, R. R. et al. Temporal dynamics of Prochlorococcus ecotypes in the Atlantic

505 and pacific oceans. ISME Journal 4, 1252-1264 (2010).

506 24. Moore, L. R. \& Chisholm, S. W. Photophysiology of the marine cyanobacterium Prochlorococcus: Ecotypic differences among cultured isolates. Limnology and Oceanography 44, 628-638 (1999).

25. Berthelot, H. et al. NanoSIMS single cell analyses reveal the contrasting nitrogen sources

26. Roth-Rosenberg, D. et al. Prochlorococcus cells rely on microbial interactions rather than on chlorotic resting stages to survive long-term nutrient starvation. mBio 11, 1-13 (2020).

27. Goericke, R. \& Welschmeyer, N. A. The marine prochlorophyte Prochlorococcus contributes significantly to phytoplankton biomass and primary production in the Sargasso Sea. Deep Sea Research Part I: Oceanographic Research Papers 40, 2283-2294 (1993).

28. Vaulot, D. The Cell Cycle of Phytoplankton: Coupling Cell Growth to Population Growth. Molecular Ecology of Aquatic Microbes 303-322 (1995) doi:10.1007/978-3-642-799235_17.

29. Binder, B. J., Chisholm, S. W., Olson, R. J., Frankel, S. L. \& Worden, A. Z. Dynamics of picophytoplankton, ultraphytoplankton and bacteria in the central equatorial Pacific. Deep Sea Research Part II: Topical Studies in Oceanography 43, 907-931 (1996).

30. Partensky, F., Blanchot, J., Lantoine, F., Neveux, J. \& Marie, D. Vertical structure of picophytoplankton at different trophic sites of the tropical northeastern Atlantic Ocean. Deep Sea Research Part I: Oceanographic Research Papers 43, 1191-1213 (1996).

31. Vaulot, D., Marie, D., Olson, R. J. \& Chisholm, S. W. Growth of Prochlorococcus, a photosynthetic prokaryote, in the equatorial Pacific Ocean. Science 268, 1480-1482 (1995).

32. Liu, H., Nolla, H. \& Campbell, L. Prochlorococcus growth rate and contribution to primary production in the equatorial and subtropical North Pacific Ocean. Aquatic Microbial Ecology 12, 39-47 (1997).

33. Murray, J., Leinen, M., Feely, R., Toggweiler, R. \& Wanninkhof, R. EqPac: A Process Study

34. Karl, D. M. \& Church, M. J. Microbial oceanography and the Hawaii Ocean Time-series programme. Nature Reviews Microbiology vol. 12 699-713 (2014). 
36. Shaked, Y., Kustka, A. B. \& Morel, F. M. M. A general kinetic model for iron acquisition by eukaryotic phytoplankton. Limnology and Oceanography 50, 872-882 (2005).

37. Bertilsson, S., Berglund, O., Karl, D. M. \& Chisholm, S. W. Elemental composition of marine Prochlorococcus and Synechococcus: Implications for the ecological stoichiometry of the sea. Limnol. Oceanogr 48, 1721-1731 (2003).

38. Roth-Rosenberg, D., Aharonovich, D., Omta, A. W., Follows, M. J. \& Sher, D. Dynamic macromolecular composition and high exudation rates in Prochlorococcus. Limnology and Oceanography 66, 1759-1773 (2021).

39. Grossowicz, M. et al. Prochlorococcus in the lab and in silico: The importance of representing exudation. Limnology and Oceanography 62, 818-835 (2017).

40. Wu, Z. et al. Modeling Photosynthesis and Exudation in Subtropical Oceans. Global Biogeochemical Cycles 35, e2021GB006941 (2021).

41. Bertlisson, S., Berglund, O., Pullin, M. J. \& Chisholm, S. W. Release of dissolved organic matter by Prochlorococcus. Vie et Milieu 55, 225-232 (2005).

42. Liu, H., Nolla, H. \& Campbell, L. Prochlorococcus growth rate and contribution to primary production in the equatorial and subtropical North Pacific Ocean. Aquatic Microbial Ecology 12, 39-47 (1997).

43. Callahan, B. J. et al. DADA2: High-resolution sample inference from Illumina amplicon data. Nature Methods 2016 13:7 13, 581-583 (2016).

44. Tamura, K., Stecher, G., Peterson, D., Filipski, A. \& Kumar, S. MEGA6: Molecular Evolutionary Genetics Analysis Version 6.0. Molecular Biology and Evolution 30, 27252729 (2013).

45. Platt, T., Gallegos, C. \& Harrison, W. Photoinhibition of photosynthesis in natural assemblages of marine phytoplankton. (1980).

46. Inomura, K. et al. A Mechanistic Model of Macromolecular Allocation, Elemental Stoichiometry, and Growth Rate in Phytoplankton. Frontiers in Microbiology 11, 86 (2020).

47. Healey, F. P. INTERACTING EFFECTS OF LIGHT AND NUTRIENT LIMITATION ON THE GROWTH RATE OF SYNECHOCOCCUS LINEARIS (CYANOPHYCEAE)1. Journal of Phycology 21, 134-146 (1985).

48. Laws, E. A. \& Bannister, T. T. Nutrient- and light-limited growth of Thalassiosira fluviatilis in continuous culture, with implications for phytoplankton growth in the ocean1. Limnology and Oceanography 25, 457-473 (1980). 